\title{
A Dilemma for Women: Love of Multiple Children or Deterioration of Diastolic Functions
}

\author{
Mehmet Ozgeyik ${ }^{1}$, Ozge Yildirim², Mevlut Kuyumcu², and Mehmet Astarcıŏlu ${ }^{3}$ \\ ${ }^{1}$ Eskisehir City Hospital \\ ${ }^{2}$ Affiliation not available \\ ${ }^{3}$ Dumlupinar University
}

August 17, 2020

\begin{abstract}
Echocardiography is the most widely used diagnostic tool for detecting the cardiac functional changes. Pregnancy is a dynamic process that affects cardiovascular system. Recent studies showed that increased parity may cause irreversible changes in cardiovascular system. In this study, we aimed to evaluate echocardiographic changes on women, especially grand multiparous (up to 9 parities) and great grand multiparous (more than 10 parities) women after all their pregnancies finished. This is a cross-sectional study and contains 195 women patients. Women with one delivery history was defined as primiparous (PP), 2 to 5 deliveries were defined as multiparous (MP), 5 to 9 deliveries were defined as grand multiparous (GMP) and more than 9 deliveries were defined as great grand multiparous (GGMP). The mean age was $50.6 \pm 16.3$ and mean parity was $6.5 \pm 4.2$. Spearman correlation analysis showed that diastolic dysfunction has positive correlations with parity, age, hypertension, and diabetes mellitus. ROC analysis showed that the best cut-off value of the parity number for predicting left ventricular diastolic dysfunction was 6.5 , with $66.3 \%$ sensitivity and $66.7 \%$ specificity. In present study, we showed that diastolic dysfunction statistically increases as the number of pregnancies increases. Additionally, cut of value of parity for diastolic dysfunction was 6.5 which is higher than other studies.
\end{abstract}

\section{Introduction}

Echocardiography is the most widely used diagnostic tool for detecting the cardiac functional changes[1]. New updates for calculating the cardiac ventricular functions are still continuing [2]. Systolic and diastolic functions of the heart can be affected by many variables [3, 4]. Especially systemic chronic diseases (diabetes mellitus, hypertension, hyperlipidemia...) deteriorate these functions. However, in healthy population, some situations as pregnancy may change cardiovascular mechanism.

Pregnancy is a dynamic process that affects cardiovascular system. During pregnancy maternal cardiac output, preload and maternal blood volume increase and systemic vascular resistance decreases [5]. These changes are necessary for the continuation of pregnancy and the health of the fetus. Most of the changes occur during pregnancy however, these changes return to normal after pregnancy [6].

Recent studies showed that increased parity may cause irreversible changes in cardiovascular system [7, 8]. Left ventricular diastolic functions deteriorate during pregnancy and this is associated with increased cardiovascular mortality [9-11]. Diastolic function and other cardiovascular changes tend to return to normal however as the parity increases, diastolic parameters are affected and these reversible changes may become permanent $[6,12]$.

In this study, we aimed to evaluate echocardiographic changes on women, especially grand multiparous (up to 9 parities) and great grand multiparous (more than 10 parities) women after all their pregnancies finished. 


\section{Methods}

This is a cross-sectional study and contains 195 women patients. All patients with a history of one or more delivery and above 18 years-of-age were included in this study. Patients under 18 years-of-age, history of coronary artery disease, heart failure, structural heart diseases, rhythm disorders, renal or hepatic disorders and women who are currently pregnant were excluded from the study. However, patients with a history of hypertension and diabetes mellitus that may affect left ventricular diastolic functions were included to study. Binary and multinominal logistic regression analyses were done for two of these situations

Women with one delivery history was defined as primiparous (PP), 2 to 5 deliveries were defined as multiparous (MP), 5 to 9 deliveries were defined as grand multiparous (GMP) and more than 9 deliveries were defined as great grand multiparous (GGMP).

Echocardiographic (Vivid 7 system with 3S echocardiography probe, GE Vingmed Ultrasound AS, Horten, Norway) evaluation was done by trained cardiology specialists. The evaluated parameters were peak early filling velocity during atrial systole (E), peak filling velocity during atrial systole (A), left ventricular ejection fraction (LVEF), left ventricular end-systolic diameter, left ventricular end-diastolic diameter, lateral e' velocity, lateral s' velocity, septal e' velocity, septal s' velocity, tricuspid S velocity. E / A was calculated as the ratio of E to A. E / e' ratio was calculated as the ratio of E velocity to mean e' (as average of lateral e' wave and septal e' wave).

Septal e' [?] $8 \mathrm{~cm} / \mathrm{sec}$, lateral e' [?]10 $\mathrm{cm} / \mathrm{sec}$ was evaluated as normal diastolic function. Diastolic dysfunction was determined to be septal e ' $<8 \mathrm{~cm} / \mathrm{sec}$, lateral e' $<10 \mathrm{~cm} / \mathrm{sec}$. Stage 1 diastolic dysfunction was defined as; mitral $\mathrm{E}$ and $\mathrm{A}$ wave velocity ratio $(\mathrm{E} / \mathrm{A})<0.8$, the ratio of $\mathrm{E}$ to the mean early diastolic mitral annular velocity (E/e') [?] 8. Stage 2 diastolic dysfunction was determined as E / A to be between 0.81.5 and $\mathrm{E} / \mathrm{e}$ ' ratio between 9 and 12. Stage 3 diastolic dysfunction was determined as $\mathrm{E} / \mathrm{A}$ ratio being [?] 2 and E / mean e' ratio was [?] 13. All these parameters were obtained American Society of Echocardiography and European Association of Cardiovascular Imaging (ASE/EACVI) guidelines recommendations[13].

Hypertension was defined as systolic pressure greater than $140 \mathrm{~mm} \mathrm{Hg}$ or diastolic pressure greater than $90 \mathrm{~mm} \mathrm{Hg}$ or a history of hypertension with the use of antihypertensive medication. Diabetes mellitus was defined as a fasting blood glucose level of $126 \mathrm{mg} / \mathrm{dl}$, a random glucose measurement of $200 \mathrm{mg} / \mathrm{dl}$, hemoglobin $\mathrm{A} 1 \mathrm{c}>6.5 \%$, or a previous diagnosis with any use of anti-diabetic medication.

Datas were presented as mean \pm standard deviation (SD) for continuous variables and as numbers and proportions for categorical variables. Distribution of the data for normality was tested by the Shapiro-Wilk test and homogeneity of group variances were tested by the Levene test. The t-test or Chi-square test was used for comparisons of continuous and categorical variables, respectively. For the parameters which are not normally distributed, Mann Whitney U test was used. More than two independent groups with normal distribution were compared with the ANOVA test. Binary logistic regression analysis was used to identify the associations of diastolic dysfunction presence to other variables. Multinominal regression analysis was used to evaluate the associations of diastolic dysfunction grades to other variables. The data analyses were performed with SPSS 23.0 (IBM SPSS Ver. 23.0, IBM Corp, Armonk NY, USA). A p-value of $<0.05$ was considered significant.

\section{Results}

Study population consists of 195 women with a history of at least one delivery. PM women constituted $8.2 \%(\mathrm{n}=16)$, MP women constituted $37.4 \%(\mathrm{n}=73)$, GMP women constituted $23.6 \%(\mathrm{n}=46)$ and GGMP women constituted $30.8 \%(\mathrm{n}=60)$ of the study population. The mean age was $50.6 \pm 16.3$ and mean parity was $6.5 \pm 4.2$. The characteristics of the study population were given in Table I.

The E velocity $(\mathrm{p}=0.017)$, A velocity $(\mathrm{p}=0.000)$, lateral e' velocity $(\mathrm{p}=0.000)$, lateral $\mathrm{s}$ ( $\mathrm{p}=0.027)$, septal e' $(p=0.000)$, septal s' $(p=0.000)$ and $E F(p=0.000)$ values were significantly different among all parity groups. The results were shown in Table II. Binary comparison of the study groups evaluating the echocardiographic parameters can be seen in Table III. 
Diastolic dysfunction classification was done according to the echocardiographic parameters. For the PM group, $87.5 \%(\mathrm{n}=14)$ had normal diastolic function, $6.25 \%(\mathrm{n}=1)$ had grade 1 diastolic dysfunction and $6.25 \%$ $(\mathrm{n}=1)$ had grade 2 diastolic dysfunction. For the MP women; $71.2 \%(\mathrm{n}=52)$ had normal diastolic function, $12.4 \%(\mathrm{n}=9)$ had grade 1 diastolic dysfunction and $16.4 \%(\mathrm{n}=12)$ had grade 2 diastolic dysfunction. For the GMP women; $56.5 \%(\mathrm{n}=26)$ had normal diastolic function, $10.9 \%(\mathrm{n}=5)$ had grade 1 diastolic dysfunction and $32.6 \%(\mathrm{n}=15)$ had grade 2 diastolic dysfunction. For the GGMP women; $28.6 \%(\mathrm{n}=17)$ had normal diastolic function, $33.2 \%(\mathrm{n}=20)$ had grade 1 diastolic dysfunction and $38.2 \%(\mathrm{n}=23)$ had grade 2 diastolic dysfunction (Table IV). There were no women with grade 3 diastolic dysfunction among the study population.

Spearman correlation analysis showed that diastolic dysfunction has positive correlations with parity, age, hypertension, and diabetes mellitus (Table V).

Table VI and Table VII report the findings of the binary and multinomial logistic regressions. Explanatory variables in both models are age, number of parity, hypertension and diabetes mellitus. The difference among the models stems from how the dependent variable is handled. In the binary logistic regression, dependent variables are grouped into two categories: diastolic dysfunction existence or the patient has normal diastolic function. On the other hand, multinomial logistic regression in this study separates the patients into three groups: patients without diastolic dysfunction, patients with grade 1 and grade 2 diastolic dysfunction. Both models show that only number of parity and age are statistically significant.

ROC analysis showed that the best cut-off value of the parity number for predicting left ventricular diastolic dysfunction was 6.5 , with $66.3 \%$ sensitivity and $66.7 \%$ specificity (Figure 1 ).

\section{Discussion}

In present study, we showed that diastolic dysfunction statistically increases as the number of pregnancies increases. Additionally, cut of value of parity for diastolic dysfunction was 6.5 which is higher than other studies $[6,12]$.

Previous studies showed that cardiovascular mortality increases along with increased parity [7]. Changes in the renin-angiotensin-aldosterone system explain this mechanism [14]. Estrogen secreted by placenta increases the release of angiotensinogen. Angiotensinogen produces angiotensin-2 that activates the RAS system. RAS system induces sodium and water retention. As a result, increased afterload is observed along with pregnancy. Also decreased relaxin levels affect cardiovascular mortality during pregnancy [15]. However, these changes continue only with pregnancy and their effects after pregnancy are still not clear.

We also found that diastolic function deteriorates as the parity increases. Aggarwal et al, performed the first known study on this issue and they found the same results[16]. Other studies similarly showed that diastolic dysfunction increases with the parity $[6,12]$. However; they performed these studies up to 7 pregnancies (grand multiparity). In our study, women that have a history of 9 and more pregnancies (great grand multiparity) were also included. The present study has the highest number of parity in the literature.

There is lack of evidence about relationship between the parity and the severity of diastolic dysfunction. Kim et al. found that 2.5 and above parity number significantly increases the diastolic dysfunction[6]. Other study performed by Keskin et al. showed that pregnancies of 4 and above significantly increase the diastolic dysfunction [12]. In our study, the cut-off value for diastolic dysfunction severity according to the ROC curve analysis was 6.5 pregnancies. This difference attributed to the number of pregnancies included in this study is higher than other studies before.

Aortic stiffness is a prognostic risk factor for cardiovascular mortality. In the present study, binary logistic regression analysis showed that presence of hypertension and diabetes mellitus did not make a significant difference in terms of diastolic dysfunction; however number of pregnancies and age did make a significant difference. This can be attributed to increasing of aortic stiffness. In our study, we showed the same results as the previous studies in the literature [17-20].

In the present study, significant decrease in ejection fraction was observed as the number of pregnancies 
increases. However, this decrease did not reach the systolic dysfunction range (greater than \%50). Although Kim et al. found same results as ours; other studies did not show this correlation $[6,12,16]$. This result attributed to be exposed of long time diastolic dysfunction as our study population has higher pregnancy numbers.

\section{LIMITATIONS}

This study has limitations that should be considered. First of all, our study population consists of limited number of patients. Second, our findings do not represent healthy population, because our study only studied patients that referred to the cardiology clinic with cardiac complaints. Third, we performed this study in a lower socio-economic area that it affects cardiovascular status independently. Finally, due to lack of facility, we did not perform cardiac magnetic resonance measurements or measure brain natriuretic peptides that show more proper information about diastolic dysfunction.

\section{Author contributions}

Concept/design: MO

Data analysis/interpretation: MO, OTY, MSK

Drafting article: MO, OTY, MSK

Critical revision of article: MO, MAA

Statistics: MO, MAA

Data collection: MO

\section{References}

1. Wood PW, Choy JB, Nanda NC, et al. Left ventricular ejection fraction and volumes: it depends on the imaging method. Echocardiography. 2014;31(1):87-100.

2. Urbano-Moral JA, Patel AR, Maron MS, et al. Three-dimensional speckle-tracking echocardiography: methodological aspects and clinical potential. Echocardiography. 2012;29(8):997-1010.

3. Ponikowski P, Voors A, Anker S, et al. Authors/Task Force Members; Document Reviewers (2016) 2016 ESC Guidelines for the diagnosis and treatment of acute and chronic heart failure: The Task Force for the diagnosis and treatment of acute and chronic heart failure of the European Society of Cardiology (ESC). Developed with the special contribution of the Heart Failure Association (HFA) of the ESC. Eur J Heart Fail. 2016;18(8):891-975.

4. Jeong E-M, Dudley Jr SC. Diastolic dysfunction. Circulation Journal. 2015;79(3):470-7.

5. Clapp III JF, Seaward BL, Sleamaker RH, et al. Maternal physiologic adaptations to early human pregnancy. American journal of obstetrics and gynecology. 1988;159(6):1456-60.

6. Kim H-J, Kim M-A, Kim H-L, et al. Effects of multiparity on left ventricular diastolic dysfunction in women: cross-sectional study of the KoRean wOmen'S chest pain rEgistry (KoROSE). BMJ open. $2018 ; 8(12)$.

7. Peters SA, Yang L, Guo Y, et al. Pregnancy, pregnancy loss, and the risk of cardiovascular disease in Chinese women: findings from the China Kadoorie Biobank. BMC medicine. 2017;15(1):148.

8. Ness RB, Harris T, Cobb J, et al. Number of pregnancies and the subsequent risk of cardiovascular disease. New England Journal of Medicine. 1993;328(21):1528-33.

9. Kuznetsova T, Herbots L, Lopez B, et al. Prevalence of left ventricular diastolic dysfunction in a general population. Circulation: Heart Failure. 2009;2(2):105-12. 
10. Mesa A, Jessurun C, Hernandez A, et al. Left ventricular diastolic function in normal human pregnancy. Circulation. 1999;99(4):511-7.

11. Moran AM, Colan SD, Mauer MB, et al. Adaptive mechanisms of left ventricular diastolic function to the physiologic load of pregnancy. Clinical Cardiology: An International Indexed and Peer-Reviewed Journal for Advances in the Treatment of Cardiovascular Disease. 2002;25(3):124-31.

12. Keskin M, Avşar Ş, Hayıroğlu Mi, et al. Relation of the number of parity to left ventricular diastolic function in pregnancy. The American Journal of Cardiology. 2017;120(1):154-9.

13. Nagueh SF, Smiseth OA, Appleton CP, et al. Recommendations for the evaluation of left ventricular diastolic function by echocardiography: an update from the American Society of Echocardiography and the European Association of Cardiovascular Imaging. European Journal of Echocardiography. 2016;17(12):132160.

14. Irani RA, Xia Y, editors. Renin angiotensin signaling in normal pregnancy and preeclampsia. Seminars in nephrology; 2011: Elsevier.

15. Teichman SL, Unemori E, Teerlink JR, et al. Relaxin: review of biology and potential role in treating heart failure. Current heart failure reports. 2010;7(2):75-82.

16. Aggarwal SR, Herrington DM, Vladutiu CJ, et al. Higher number of live births is associated with left ventricular diastolic dysfunction and adverse cardiac remodelling among US Hispanic/Latina women: results from the Echocardiographic Study of Latinos. Open Heart. 2017;4(1).

17. Mahendru AA, Everett TR, Wilkinson IB, et al. A longitudinal study of maternal cardiovascular function from preconception to the postpartum period. Journal of hypertension. 2014;32(4):849-56.

18. Fujime M, Tomimatsu T, Okaue $\mathrm{Y}$, et al. Central aortic blood pressure and augmentation index during normal pregnancy. Hypertension Research. 2012;35(6):633-8.

19. Mitchell GF, Guo C-Y, Benjamin EJ, et al. Cross-sectional correlates of increased aortic stiffness in the community. Circulation. 2007;115:2628-36.

20. Ungvari Z, Kaley G, de Cabo R, et al. Mechanisms of vascular aging: new perspectives. Journals of Gerontology Series A: Biomedical Sciences and Medical Sciences. 2010;65(10):1028-41.

Table I. Characteristics of the study population

\begin{tabular}{|c|c|c|c|c|c|}
\hline & $\begin{array}{l}\text { Primiparous } 1 \\
\text { delivery } \mathrm{n}=16\end{array}$ & $\begin{array}{l}\text { Multiparous } 1< \\
\text { to } 5 \text { deliveries } \\
\mathrm{n}=73\end{array}$ & $\begin{array}{l}\text { Grand } \\
\text { multiparous } 5< \\
\text { to } 9 \text { deliveries } \\
\mathrm{n}=46\end{array}$ & $\begin{array}{l}\text { Great grand } \\
\text { multiparous } 9< \\
\text { deliveries } \mathrm{n}=60\end{array}$ & $\mathrm{p}$ \\
\hline $\begin{array}{l}\text { Age, years } \\
( \pm \mathrm{SD})\end{array}$ & $30.8 \pm 9.0$ & $39.4 \pm 8.8$ & $52.8 \pm 11.9$ & $67.7 \pm 9.5$ & 0.000 \\
\hline $\begin{array}{l}\text { Parity } \\
\text { number, n }\end{array}$ & $1.0 \pm 0.0$ & $2.9 \pm 1.0$ & $7.5 \pm 1.2$ & $11.7 \pm 1.8$ & 0.000 \\
\hline $\begin{array}{l}\text { Diabetes } \\
\text { mellitus, n (\%) }\end{array}$ & $2,12.5 \%$ & $3,4.1 \%$ & $8,17.4 \%$ & $19,31.7 \%$ & 0.000 \\
\hline $\begin{array}{l}\text { Hypertension, } \\
\mathrm{n}(\%)\end{array}$ & $4,25.0 \%$ & $19,26.0 \%$ & $32,69.6 \%$ & $55,91.6 \%$ & 0.000 \\
\hline $\begin{array}{l}\text { Sistolic BP, } \\
\mathrm{mmHg}\end{array}$ & $105.6 \pm 15.9$ & $115.6 \pm 19.4$ & $132.4 \pm 22.0$ & $137.5 \pm 23.1$ & 0.000 \\
\hline $\begin{array}{l}\text { Diastolic BP, } \\
\mathrm{mmHg}\end{array}$ & $67.1 \pm 9.6$ & $75.5 \pm 12.0$ & $83.0 \pm 11.3$ & $83.2 \pm 12.9$ & 0.000 \\
\hline
\end{tabular}


Abb. BP, blood pressure; SD, standard deviation.

Table II. Echocardiographic parameters of the study groups

\begin{tabular}{llllll}
\hline Variables & PP & MP & GMP & GGMP & p \\
\hline E, cm/s & $87.7 \pm 15.4$ & $89.1 \pm 26.2$ & $102.4 \pm 19.8$ & $90.7 \pm 25.3$ & $\mathbf{0 . 0 1 7}$ \\
A, cm/s & $66.6 \pm 11.8$ & $78.2 \pm 20.4$ & $101.6 \pm 21.4$ & $101.6 \pm 20.1$ & $\mathbf{0 . 0 0 0}$ \\
Lateral e', cm/sn & $14.0 \pm 3.3$ & $12.7 \pm 3.4$ & $10.6 \pm 3.0$ & $8.6 \pm 2.7$ & $\mathbf{0 . 0 0 0}$ \\
Lateral s', cm/sn & $10.1 \pm 0.9$ & $10.3 \pm 2.0$ & $9.7 \pm 2.1$ & $9.3 \pm 2.4$ & $\mathbf{0 . 0 2 7}$ \\
Septal e', cm/sn & $10.5 \pm 2.3$ & $9.5 \pm 2.4$ & $7.4 \pm 2.1$ & $6.2 \pm 1.7$ & $\mathbf{0 . 0 0 0}$ \\
Septal s', cm/sn & $8.1 \pm 1.1$ & $8.2 \pm 1.5$ & $8.1 \pm 2.0$ & $7.0 \pm 1.8$ & $\mathbf{0 . 0 0 0}$ \\
EDD, mm & $44.7 \pm 2.4$ & $44.4 \pm 3.5$ & $44.8 \pm 4.4$ & $44.5 \pm 6.0$ & 0.964 \\
ESD, mm & $28.7 \pm 2.3$ & $27.4 \pm 3.8$ & $27.1 \pm 4.9$ & $27.2 \pm 6.5$ & 0.733 \\
EF, \% & $62.3 \pm 3.1$ & $62.4 \pm 3.0$ & $58.9 \pm 5.4$ & $56.2 \pm 6.7$ & $\mathbf{0 . 0 0 0}$ \\
\hline
\end{tabular}

Table III. Binary comparison of parity groups according to parameters

\begin{tabular}{lllllll}
\hline & PP vs MP & PP vs GMP & PP vs GGMP & MP vs GMP & MP vs GGMP & GMP vs GGMP \\
\hline E & 0.844 & 0.009 & 0.779 & $\mathbf{0 . 0 0 4}$ & 0.714 & 0.011 \\
A & 0.025 & $\mathbf{0 . 0 0 0}$ & $\mathbf{0 . 0 0 0}$ & $\mathbf{0 . 0 0 0}$ & $\mathbf{0 . 0 0 0}$ & $\mathbf{0 . 9 8 7}$ \\
Lateral e' & 0.147 & $\mathbf{0 . 0 0 0}$ & $\mathbf{0 . 0 0 0}$ & $\mathbf{0 . 0 0 1}$ & $\mathbf{0 . 0 0 0}$ & $\mathbf{0 . 0 0 0}$ \\
Lateral s' & 0.623 & 0.442 & $\mathbf{0 . 0 2 6}$ & 0.084 & $\mathbf{0 . 0 0 5}$ & 0.350 \\
Septal e' & 0.138 & $\mathbf{0 . 0 0 0}$ & $\mathbf{0 . 0 0 0}$ & $\mathbf{0 . 0 0 0}$ & $\mathbf{0 . 0 0 0}$ & $\mathbf{0 . 0 0 1}$ \\
Septal s' & 0.830 & 0.907 & $\mathbf{0 . 0 1 8}$ & 0.645 & $\mathbf{0 . 0 0 0}$ & $\mathbf{0 . 0 0 3}$ \\
EF & 0.892 & $\mathbf{0 . 0 1 9}$ & $\mathbf{0 . 0 0 0}$ & $\mathbf{0 . 0 0 0}$ & $\mathbf{0 . 0 0 0}$ & $\mathbf{0 . 0 2 7}$ \\
\hline
\end{tabular}

Table IV. Diastolic function classification among study population

\begin{tabular}{lllll}
\hline & $\mathrm{PP}(\mathrm{n}=16)$ & $\mathrm{MP}(\mathrm{n}=73)$ & $\mathrm{GMP}(\mathrm{n}=46)$ & $\operatorname{GGMP}(\mathrm{n}=60)$ \\
\hline Normal diastolic function & 14 & 52 & 26 & 17 \\
DD Grade 1 & 1 & 9 & 5 & 20 \\
DD Grade 2 & 1 & 12 & 15 & 23 \\
\hline
\end{tabular}

Table V. Spearman correlation analysis between the diastolic dysfunction presence and number of parity, age, hypertension and diabetes mellitus

\begin{tabular}{lllll}
\hline & Number of parity & Age & Hypertension & Diabetes Mellitus \\
\hline $\mathrm{r}$ & 0.404 & 0.614 & 0.448 & 0.331 \\
$\mathrm{p}$ & $\mathbf{0 . 0 0 0}$ & $\mathbf{0 . 0 0 0}$ & $\mathbf{0 . 0 0 0}$ & $\mathbf{0 . 0 0 0}$ \\
\hline
\end{tabular}

Table VI. Binary logistic regression analysis for the presence of diastolic dysfunction

\begin{tabular}{llll}
\hline & Odds Ratio & $95 \%$ CI & P \\
\hline Number of parity & 0.805 & $0.692-0.938$ & $\mathbf{0 . 0 0 5}$ \\
Age & 1.166 & $1.103-1.234$ & $\mathbf{0 . 0 0 0}$
\end{tabular}




\begin{tabular}{llll}
\hline & Odds Ratio & $95 \%$ CI & P \\
\hline Hypertension & 0.968 & $0.359-2.611$ & 0.949 \\
Diabetes Mellitus & 0.520 & $0.176-1.536$ & 0.237 \\
\hline
\end{tabular}

Table VII. Multinomial logistic regression analysis for diastolic dysfunction grade

\begin{tabular}{lllll}
\hline Diastolic Dysfunction Grades & & Odds ratio & $95 \%$ & $\mathrm{p}$ \\
\hline Grade 1 & Number of parity & 0.760 & $0.625-0.925$ & $\mathbf{0 . 0 0 6}$ \\
& Age & 1.198 & $1.118-1.283$ & $\mathbf{0 . 0 0 0}$ \\
& Hypertension & 0.885 & $0.217-3.614$ & 0.865 \\
Grade 2 & Diabetes Mellitus & 0.525 & $0.152-1.815$ & 0.309 \\
& Number of parity & 0.829 & $0.705-0.976$ & $\mathbf{0 . 0 2 4}$ \\
& Age & 1.150 & $1.084-1.220$ & $\mathbf{0 . 0 0 0}$ \\
& Hypertension & 1.006 & $0.334-3.032$ & 0.991 \\
& Diabetes mellitus & 0.516 & $0.164-1.621$ & 0.257 \\
\hline
\end{tabular}

Figure I. Receiver-operating curve (ROC) analysis. ROC analysis revealed that the best cut-off value of the parity number for predicting left ventricular diastolic dysfunction was 6.5 , with $66.3 \%$ sensitivity and $66.7 \%$ specificity (Area Under the Curve: $0.734 ; 95 \%$ CI 0.663 to $0.805 ; \mathrm{p}<0.000$ ). 


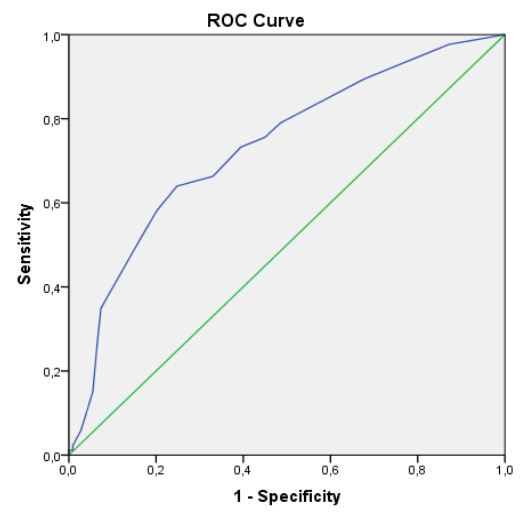

